\title{
ON THE FAILURE OF GRANULAR MATERIALS WITH FABRIC EFFECTS
}

\author{
Pei J. Guoi) and Dieter F. E. Stolle ${ }^{\text {ii) }}$
}

\begin{abstract}
Anisotropic failure criteria for granular materials are formulated, based on micromechanical analysis. The shear resistance is assumed to consist of two components related to fabric anisotropy and interparticle frictional forces. By considering the directional contribution of contact normals, the failure criteria are developed via two approaches: one employs the invariants of stress and fabric tensors; and the other considers the relation between the traction components acting on the critical plane. In the stress invariant approach, however, some microscopic features of granular materials may not be captured due to the utilization of stress invariants. Numerical examples, examining the variation of shear strength of granular materials with different orientations of bedding plane and the degree of anisotropy, compare the proposed approaches with other models in the literature.
\end{abstract}

Key words: fabric, failure criterion, granular material, micro-mechanics (IGC: D6)

\section{INTRODUCTION}

Numerous experimental studies show that the failure of granular soils is intrinsically related to internal structures; see, for example, Biarez and Wiendieck (1963), Oda and Konishi (1974), Oda et al. (1978), Oda et al. (1985), Tatsuoka et al. (1986), Yoshimine and Ishihara (1998) and Vaid and Saivathayalan (2000). Thus, it is pertinent to study the effect of fabric in sand and systematically incorporate its influence in constitutive models when dealing with the analysis of failure, material instability, as well as strain localization.

A rigorous approach to identify failure makes use of the general representation theorems that employ invariants of stress and structure-orientation or fabric tensors as described by Nova (1980), Rogers (1987), Boehler (1987) and Oda (1989). According to Oda et al. (1985), the anisotropy of granular materials stems from three sources, including: preferred orientation of contact normals; preferred alignment of non-spherical particles; and the elongated shape of voids. Quantities related to these sources have been used to characterize the fabric of granular materials; see, e.g., Satake (1982), Chang and Misra (1988), Kuo and Frost (1996), Muhunthan et al. (1996), Emeriault and Chang (1997) and Pietruszczak (1999).

Failure criteria of anisotropic geomaterials have also been derived through the critical plane approach. Having been used for rock masses for many years (e.g., Walsh and Brace, 1964; Hoek and Brown, 1980), this approach assumes that failure occurs along a plane (i.e., the critical plane) on which the failure criterion is first satisfied. The critical plane orientation is usually determined by maximizing some failure function in terms of normal and tangential components of the traction vector for all potential failure planes. More recently, Pietruszczak and Mroz (2001) applied this approach to anisotropic soils by incorporating a distribution of strength parameters dependent on orientation. Based on microscopic analysis, Nemat-Nasser (2000) proposed that the shear resistance on a critical failure plane consists of components associated with interparticle friction and fabric anisotropy, respectively. He quantifies the effect of fabric by a second-rank tensor that describes the distribution of contact normals and assumes that failure takes place on a critical plane corresponding to the maximum of some failure function.

For granular materials composed of distinct particles interacting at contact points, however, their mechanical behaviours are significantly influenced by the spatial arrangement of particles, as well as the internal constraints due to the geometry and connectivity of particles. Moreover, the local behaviour of granular materials at a contact point is primarily ruled by the orientation of the contact plane together with the local shear and normal contact forces. Constitutive models based on macromechanical observations when granular materials are considered as continua on the macroscopic scale may not be able to capture the discrete features appropriately. Development in the micromechanics of granular materials has provided useful tools for the quantification of fabric as well as the relations between micro- and

i) Asst. Professor, Department of Civil Engineering, McMaster University, 1280 Main Street West, Hamilton, Ontario, Canada L8S 4L7 (guop @mcmaster.ca)

ii) Professor, ditto (stolle@mcmaster.ca).

The manuscript for this paper was received for review on March 5, 2004; approved on March 11, 2005.

Written discussions on this paper should be submitted before March 1, 2006 to the Japanese Geotechnical Society, 4-38-2, Sengoku, Bunkyo$\mathrm{ku}$, Tokyo 112-0011, Japan. Upon request the closing date may be extended one month. 
macro-quantities such as interparticle forces and average stresses, relative displacement between particles and average strains. As a consequence, it is possible to develop an alternative micro-macro mechanical approach to deduce the behaviour of the equivalent continuum with micromechanical considerations to accommodate the discrete features of granular materials more appropriately. Details can be found in Chang (1993), Cambou et al. (1995) and Emeriault and Chang (1997) among others.

These considerations provide the impetus for the present paper, which addresses the failure mechanism of anisotropic granular materials and derives a failure criterion based on micromechanical considerations. For a deforming particulate ensemble, the stress is decomposed into three parts, namely, an isotropic component associated with normal interparticle forces, a deviatoric component related to fabric, and a deviatoric component induced by interparticle frictional forces. Making use of this decomposition, a failure criterion is identified that includes the orientational distribution of contact normals. A discussion of the proposed approaches and the models developed by Pietruszczak and Mroz (2001) and Nemat-Nasser (2000) is provided. The performances of these formulations are illustrated and compared by a series of numerical examples that examine the variation of shear strength of granular materials with different orientations of bedding planes.

\section{STATICS AND AVERAGE STRESS}

\section{Average Stress in Terms of Contact Forces}

According to Landau and Lifshitz (1959), for the case of discrete point loads applied at a boundary of a material element, the volume average of the stress tensor $\langle\sigma\rangle$ is related to boundary forces $\mathbf{f}^{\mathrm{b}}$ via

$$
\left\langle\sigma_{\mathrm{ij}}\right\rangle=\frac{1}{V} \int_{V} \sigma_{\mathrm{ij}} d V=\frac{1}{V} \sum_{b \in S}^{n_{\mathrm{b}}} x_{\mathrm{i}}^{\mathrm{b}} f_{\mathrm{j}}^{\mathrm{b}}
$$

where the subscript and the superscript $b$ denote a quantity on the element boundary $S, \mathbf{x}^{\mathrm{b}}$ are locations of points where the $\mathbf{f}^{\mathrm{b}}$ are applied, and $n_{\mathrm{b}}$ is the number of contacts on $S$. Equation (1) may be obtained by considering equilibrium of a weightless body for which $\sigma_{\mathrm{kj}, \mathrm{k}}=0$ and applying Gauss integral formula. It should be noted that the average stress tensor $\left\langle\sigma_{\mathrm{ij}}\right\rangle$ is not necessarily symmetrical by virtue of the non-commutativity of the product $x_{\mathrm{i}}^{\mathrm{b}} f_{\mathrm{j}}^{\mathrm{b}}$. The physical meaning of Eq. (1) can be easily interpreted for a $2 \mathrm{D}$ rectangular sample. In this case, Eq. (1) is in fact a statement of force balance condition for the element with no body forces. Even though Eq. (1) originates in continuum mechanics, it can be applied to an ensemble of discrete particles provided that the representative volume element (RVE) contains a sufficiently large number of particles.

\section{Average Stress in Terms of Contact Forces}

When body forces are negligible, the equilibrium condition for a typical particle $\alpha$ (see Fig. 1) is given by

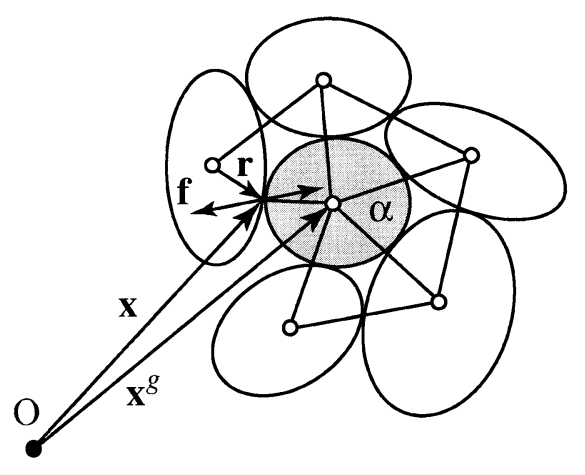

Fig. 1. Description of grain topology

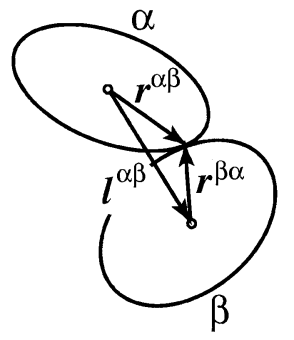

(a)

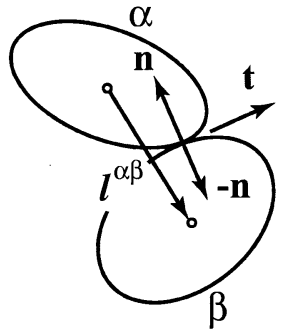

(b)
Fig. 2. Definition of (a) branch vector and (b) contact normals

$$
\sum_{\beta}^{C} f_{\mathrm{j}}^{\beta}=0
$$

where $C$ is the number of contacts over particle $\alpha$ with $j \in[1,2,3]$. Multiplication of Eq. (2) by the position vector component $x_{\mathrm{i}}^{\mathrm{g} \alpha}$ of particle $\alpha$ with $i \in[1,2,3]$ yields

$$
x_{\mathrm{i}}^{\mathrm{g} \alpha} \sum_{\beta}^{C} f_{\mathrm{j}}^{\beta}=\sum_{\beta}^{C}\left(x_{\mathrm{i}}^{\beta}-r_{\mathrm{i}}^{\beta}\right) f_{\mathrm{j}}^{\beta}=0
$$

The equilibrium condition for all particles can be expressed as

$$
\sum_{\alpha}^{n_{g}} \sum_{\beta}^{C}\left(x_{\mathrm{i}}^{\beta}-r_{\mathrm{i}}^{\beta}\right) f_{\mathrm{j}}^{\beta}=0 \quad \text { or } \quad \sum_{\alpha}^{n_{g}} \sum_{\beta}^{C} x_{\mathrm{i}}^{\beta} f_{\mathrm{j}}^{\beta}=\sum_{\alpha}^{n_{g}} \sum_{\beta}^{C} r_{\mathrm{i}}^{\beta} f_{\mathrm{j}}^{\beta}
$$

Referring to Fig. 1 and invoking Newton's third law, the $x_{\mathrm{i}} f_{\mathrm{j}}$ terms at all internal contact points cancel out, resulting in

$$
\begin{gathered}
\sum_{\alpha}^{n_{g}} \sum_{\beta}^{C} x_{\mathrm{i}}^{\beta} f_{\mathrm{j}}^{\beta}=\sum_{b \in S}^{n_{b}} x_{\mathrm{i}}^{\mathrm{b}} f_{\mathrm{j}}^{\mathrm{b}} \\
\sum_{\alpha}^{n_{g}} \sum_{\beta}^{C} r_{\mathrm{i}}^{\alpha} f_{\mathrm{j}}^{\beta}=\sum^{n_{c}}\left(r_{\mathrm{i}}^{\alpha \beta} f_{\mathrm{j}}^{\alpha \beta}+r_{\mathrm{i}}^{\beta \alpha} f_{\mathrm{j}}^{\beta \alpha}\right)
\end{gathered}
$$

where the double superscript $\alpha \beta$ denotes the quantity associated with grain $\alpha$ at contact point $\beta$ and $n_{\mathrm{c}}$ is the total number of contacts in the RVE. By introducing a branch vector $l_{\mathrm{i}}^{\alpha \beta}$ which connects the centers of gravity of two contacting grains as shown in Fig. 2 such that $l_{\mathrm{i}}^{\alpha \beta}=r_{\mathrm{i}}^{\alpha \beta}-r_{\mathrm{i}}^{\beta \alpha}$, some measure of the internal structure (grain arrangement) can be defined. By recognizing that $f_{\mathrm{j}}^{\beta \alpha}=-f_{\mathrm{j}}^{\alpha \beta}$ due to equilibrium, one has 


$$
\begin{aligned}
\sum_{b \in S}^{n_{b}} x_{\mathrm{i}}^{\mathrm{b}} f_{\mathrm{j}}^{\mathrm{b}} & =\sum^{n_{c}}\left(r_{\mathrm{i}}^{\alpha \beta} f_{\mathrm{j}}^{\alpha \beta}+r_{\mathrm{i}}^{\beta \alpha} f_{\mathrm{j}}^{\beta \alpha}\right)=\sum^{n_{c}}\left(r_{\mathrm{i}}^{\alpha \beta}-r_{\mathrm{i}}^{\beta \alpha}\right) f_{\mathrm{j}}^{\alpha \beta} \\
& =\sum^{n_{c}} l_{\mathrm{i}}^{\alpha \beta} f_{\mathrm{j}}^{\alpha \beta}
\end{aligned}
$$

and Eq. (1) becomes

$$
\left\langle\sigma_{\mathrm{ij}}\right\rangle=\frac{1}{V} \sum^{n_{c}} l_{\mathrm{i}}^{\alpha \beta} f_{\mathrm{j}}^{\alpha \beta}=\frac{1}{V} \sum^{n_{c}} \mathbf{1} \otimes \mathbf{f}
$$

with $\otimes$ denoting an outer-product operation. Equation (8) represents an expression of the average stress tensor in terms of particle contact forces and a fabric measure that enters via the branch vector $\mathbf{l}$.

\section{Decomposition of Average Stress Tensor}

In general, the contact force $\mathbf{f}$ can be decomposed as

$$
\mathbf{f}=f_{N} \mathbf{n}+f_{\mathrm{t}} \mathbf{t}
$$

where $f_{\mathrm{N}}$ and $f_{\mathrm{t}}$ are the contact force components corresponding to the unit contact normal $\mathbf{n}$ and tangent $\mathbf{t}$, respectively, as shown in Fig. 2. Substituting Eq. (9) into Eq. (8) yields (Thornton, 2000)

$$
\left\langle\sigma_{\mathrm{ij}}\right\rangle=\left\langle\sigma_{\mathrm{ij}}^{\mathrm{N}}\right\rangle+\left\langle\sigma_{\mathrm{ij}}^{\mathrm{T}}\right\rangle
$$

with

$$
\left\langle\sigma_{\mathrm{ij}}^{\mathrm{N}}\right\rangle=\frac{1}{V} \sum_{1}^{n_{c}} f_{\mathrm{N}} \mathbf{I} \otimes \mathbf{n} ; \quad\left\langle\sigma_{\mathrm{ij}}^{\mathrm{T}}\right\rangle=\frac{1}{V} \sum_{1}^{n_{c}} f_{\mathrm{t}} \mathbf{I} \otimes \mathbf{t}
$$

being the normal and tangential contact force contributions to the average stress tensor, respectively.

Without loss of generality, let us now consider twodimensional stress conditions and assume that all particles are spheres with radius $R$. For this particular case, one has for example

$$
\left\langle\sigma_{\mathrm{ij}}^{\mathrm{N}}\right\rangle=\frac{2 R}{V} \sum_{1}^{n_{c}} f_{\mathrm{N}} n_{\mathrm{i}} n_{\mathrm{j}}, \quad\left\langle\sigma_{\mathrm{ij}}^{\mathrm{T}}\right\rangle=\frac{2 R}{V} \sum_{1}^{n_{c}} f_{\mathrm{t}} n_{\mathrm{i}} t_{\mathrm{j}}
$$

with the branch vector $\mathbf{I}=2 R \mathbf{n}$. By invoking

$$
f_{\mathrm{N}}=\mathbf{f} \cdot \mathbf{n}=f_{\mathrm{k}} n_{\mathrm{k}}
$$

the expression for $\left\langle\sigma_{\mathrm{ij}}^{\mathrm{N}}\right\rangle$ becomes

$$
\left\langle\sigma_{\mathrm{ij}}^{\mathrm{N}}\right\rangle=\frac{2 R}{V} \sum_{1}^{n_{c}} f_{\mathrm{N}} n_{\mathrm{i}} n_{\mathrm{j}}=\frac{2 R}{V} \sum_{1}^{n_{c}} f_{\mathrm{k}} n_{\mathrm{k}} n_{\mathrm{i}} n_{\mathrm{j}}
$$

Substituting Eq. (14) into Eq. (10) yields

$$
\left\langle\sigma_{\mathrm{ij}}^{\mathrm{T}}\right\rangle=\left\langle\sigma_{\mathrm{ij}}\right\rangle-\left\langle\sigma_{\mathrm{ij}}^{\mathrm{N}}\right\rangle=\frac{2 R}{V} \sum_{1}^{n_{c}} n_{\mathrm{i}} f_{\mathrm{j}}-\frac{2 R}{V} \sum_{1}^{n_{c}}\left(f_{\mathrm{k}} n_{\mathrm{k}}\right) n_{\mathrm{i}} n_{\mathrm{j}}
$$

and

$$
\left\langle\sigma_{\mathrm{ii}}^{\mathrm{T}}\right\rangle=\frac{2 R}{V} \sum_{1}^{n_{c}} n_{\mathrm{i}} f_{\mathrm{i}}-\frac{2 R}{V} \sum_{1}^{n_{c}} f_{\mathrm{k}} n_{\mathrm{k}} n_{\mathrm{i}} n_{\mathrm{i}}=0
$$

which indicates that the stress tensor $\left\langle\sigma_{\mathrm{ij}}^{\mathrm{T}}\right\rangle$ is traceless. For two-dimensional stress conditions, the spherical component of $\left\langle\sigma_{\mathrm{ij}}\right\rangle$ is then

$$
\begin{aligned}
S= & \frac{1}{2}\left\langle\sigma_{\mathrm{kk}}\right\rangle=\frac{1}{2}\left\langle\sigma_{\mathrm{kk}}^{\mathrm{N}}\right\rangle=\frac{1}{2} \frac{2 R}{V} \sum_{1}^{n_{c}} f_{\mathrm{N}} n_{\mathrm{i}} n_{\mathrm{i}} \\
= & \frac{1}{2} \frac{2 R}{V} \sum_{1}^{n_{c}}\left\langle f_{\mathrm{N}}\right\rangle n_{\mathrm{i}} n_{\mathrm{i}}=\frac{R n_{c}}{V}\left\langle f_{\mathrm{N}}\right\rangle
\end{aligned}
$$

where $\left\langle f_{\mathrm{N}}\right\rangle$ is the average of the normal component of contact forces. The average $\left\langle\sigma_{\mathrm{ij}}^{\mathrm{N}}\right\rangle$ can be expressed as

$$
\left\langle\sigma_{\mathrm{ij}}^{\mathrm{N}}\right\rangle=\frac{2 R}{V} \sum_{1}^{n_{c}}\left\langle f_{\mathrm{N}}\right\rangle n_{\mathrm{i}} n_{\mathrm{j}}=\left\langle\sigma_{\mathrm{kk}}\right\rangle\left\langle n_{\mathrm{i}} n_{\mathrm{j}}\right\rangle
$$

As a result, Eq. (10) becomes

$$
\left\langle\sigma_{\mathrm{ij}}\right\rangle=\left\langle\sigma_{\mathrm{kk}}\right\rangle\left\langle n_{\mathrm{i}} n_{\mathrm{j}}\right\rangle+\left\langle\sigma_{\mathrm{ij}}^{\mathrm{T}}\right\rangle
$$

The introduction of a second-rank fabric tensor (Satake, 1982)

$$
F_{\mathrm{ij}}=\left\langle n_{\mathrm{i}} n_{\mathrm{j}}\right\rangle=\frac{1}{n_{c}} \sum_{\mathrm{k}=1}^{n_{c}} n_{\mathrm{i}}^{\mathrm{k}} n_{\mathrm{j}}^{\mathrm{k}} ; \quad F_{\mathrm{ii}}=1
$$

leads to

$$
\left\langle\sigma_{\mathrm{ij}}^{\mathrm{N}}\right\rangle=\left\langle\sigma_{\mathrm{kk}}\right\rangle F_{\mathrm{ij}}
$$

One finds that $\left\langle\sigma_{\mathrm{ij}}^{\mathrm{N}}\right\rangle$ is coaxial with the fabric tensor $F_{\mathrm{ij}}$.

In general, the distribution of the unit branch vector (or the unit contact normals) can be described by a density function (Kanatani, 1984; Cowin, 1985)

$$
E(\mathbf{n})=\frac{1}{2 \pi}\left(\omega_{\mathrm{ij}}+\delta_{\mathrm{ij}}\right) n_{\mathrm{i}} n_{\mathrm{j}}
$$

where $\omega$ is a symmetric traceless second-rank tensor, and $\delta_{\mathrm{ij}}$ is Kronecker delta with $\delta_{\mathrm{ii}}=2$ for two-dimensional cases. Equation (21) may be rewritten as (Rothenburg and Bathurst, 1989)

$$
E(\mathbf{n})=\frac{1}{2 \pi}\left[1+\bar{\omega} \cos 2\left(\theta-\theta_{0}\right)\right]
$$

where the second invariant of tensor $\omega$

$$
\bar{\omega}=\left(\frac{1}{2} \omega_{\mathrm{ij}} \omega_{\mathrm{ij}}\right)^{1 / 2}
$$

defines the degree of anisotropy according to Eq. (21) and $\theta_{0}$ gives the orientation of the greatest density of the branch vector (or the contact normals); i.e. the major principal direction of tensor $\omega$. If one further expresses the fabric tensor as (Satake, 1982)

$$
\mathbf{F}=\langle\mathbf{n} \otimes \mathbf{n}\rangle=\int_{\Omega} E(\mathbf{n}) \mathbf{n} \otimes \mathbf{n} d \Omega
$$

with $\langle\bullet\rangle$ denoting the volume average and $\Omega$ a unit sphere, it follows that

$$
\omega_{\mathrm{ij}}=4\left(F_{\mathrm{ij}}-\frac{1}{2} \delta_{\mathrm{ij}}\right), F_{\mathrm{ij}}=\frac{1}{2}\left(\delta_{\mathrm{ij}}+\frac{1}{2} \omega_{\mathrm{ij}}\right)
$$

and

$$
\left\langle\sigma_{\mathrm{ij}}\right\rangle=\frac{1}{V} \int_{\Omega} E(\mathbf{n}) \mathbf{l} \otimes \mathbf{f} d \Omega
$$

with 

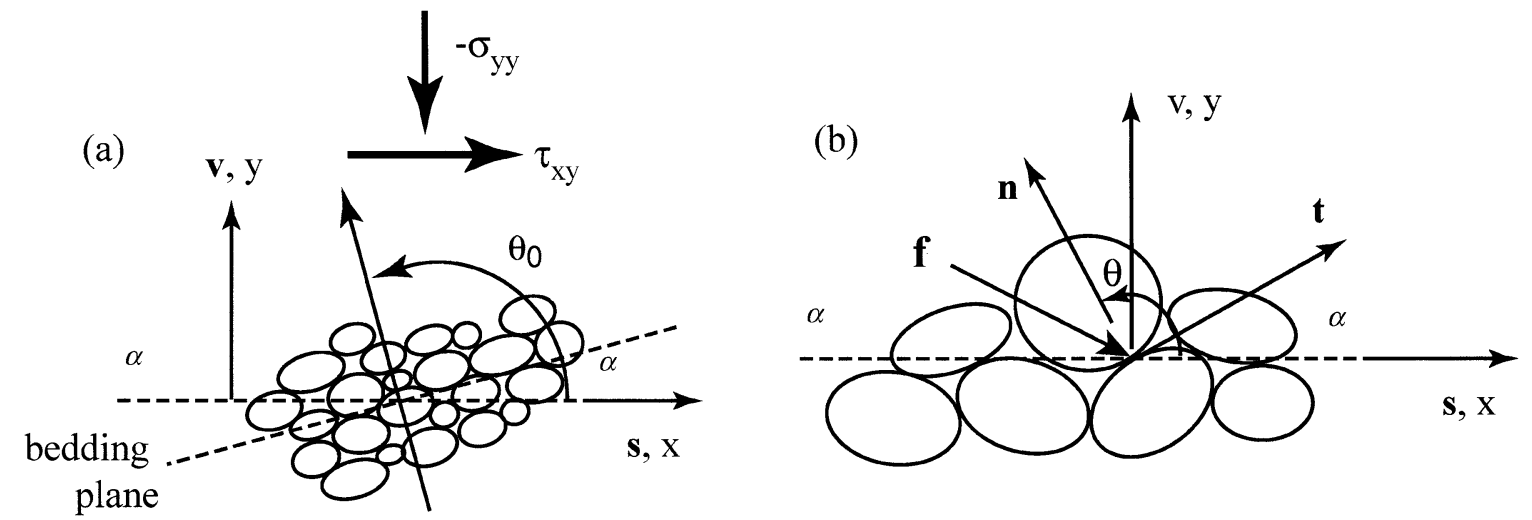

Fig. 3. Schematic representation of a macroscopic failure plane with unit normal $v$ : (a) the principal direction of fabric and bedding plane and (b) the macroscopic and the local sliding directions defined by the unit vector $s$ and $t$ respectively

$$
\begin{aligned}
& \left\langle\sigma_{\mathrm{ij}}^{\mathrm{N}}\right\rangle=\frac{2 R}{V} \int_{\Omega} f_{\mathrm{N}} E(\mathbf{n}) \mathbf{n} \otimes \mathbf{n} d \Omega, \\
& \left\langle\sigma_{\mathrm{ij}}^{\mathrm{T}}\right\rangle=\frac{2 R}{V} \int_{\Omega} f_{\mathrm{t}} E(\mathbf{n}) \mathbf{n} \otimes \mathbf{t} d \Omega
\end{aligned}
$$

The stress component $\left\langle\sigma_{\mathrm{ij}}^{\mathrm{N}}\right\rangle$ can be further decomposed as

$$
\left\langle\sigma_{\mathrm{ij}}^{\mathrm{N}}\right\rangle=\frac{\left\langle\sigma_{\mathrm{kk}}\right\rangle}{2}\left(\delta_{\mathrm{ij}}+\frac{1}{2} \omega_{\mathrm{ij}}\right)=s \delta_{\mathrm{ij}}+\left\langle\sigma_{\mathrm{ij}}^{\mathrm{f}}\right\rangle
$$

with

$$
\left\langle\sigma_{\mathrm{ij}}^{\mathrm{f}}\right\rangle=\frac{1}{2} s \omega_{\mathrm{ij}} ; \quad s=\frac{1}{2}\left\langle\sigma_{\mathrm{kk}}\right\rangle
$$

where the second-rank tensor $\left\langle\sigma_{\mathrm{ij}}^{\mathrm{f}}\right\rangle$ is traceless. Finally, the decomposition of the average stress tensor $\left\langle\sigma_{\mathrm{ij}}\right\rangle$ is given as

$$
\left\langle\sigma_{\mathrm{ij}}\right\rangle=s \delta_{\mathrm{ij}}+\left\langle\sigma_{\mathrm{ij}}^{\mathrm{f}}\right\rangle+\left\langle\sigma_{\mathrm{ij}}^{\mathrm{T}}\right\rangle
$$

At this point, it should be mentioned that $\left\langle\sigma_{\mathrm{ij}}\right\rangle,\left\langle\sigma_{\mathrm{ij}}^{\mathrm{f}}\right\rangle$ and $\left\langle\sigma_{\mathrm{ij}}^{\mathrm{T}}\right\rangle$ are not necessarily coaxial.

In continuum mechanics, $\left\langle\sigma_{\mathrm{ij}}\right\rangle$ is usually decomposed into its spherical and deviatoric components:

$$
\left\langle\sigma_{\mathrm{ij}}\right\rangle=s \delta_{\mathrm{ij}}+S_{\mathrm{ij}}
$$

A comparison of Eqs. (30) with (31) yields

$$
S_{\mathrm{ij}}=\left\langle\sigma_{\mathrm{ij}}^{\mathrm{f}}\right\rangle+\left\langle\sigma_{\mathrm{ij}}^{\mathrm{T}}\right\rangle=\frac{1}{2} s \omega_{\mathrm{ij}}+s_{\mathrm{ij}}^{\mathrm{T}}
$$

Even though $\left\langle\sigma_{\mathrm{ij}}\right\rangle$ and the deviatoric stress tensor $s_{i j}$ are always coaxial, $\omega_{i j}$ and hence $\left\langle\sigma_{\mathrm{ij}}^{\mathrm{f}}\right\rangle$ may be non-coaxial with $\left\langle\sigma_{\mathrm{ij}}\right\rangle$. This implies that $s_{\mathrm{ij}}^{\mathrm{T}}$ from the contribution of frictional components of contact forces might be noncoaxial with $\left\langle\sigma_{\mathrm{ij}}\right\rangle$ for anisotropic granular materials.

According to Eq. (11), $\left\langle\sigma_{\mathrm{ij}}^{\mathrm{N}}\right\rangle$, which is coaxial with the fabric tensor $F_{\mathrm{ij}}$, may be interpreted as the stress in a granular mass induced by purely normal contact forces. One expects that no sliding of particles occurs under $\left\langle\sigma_{\mathrm{ij}}^{\mathrm{N}}\right\rangle$ since there is no tangential force at any contact point. As such, $\left\langle\sigma_{\mathrm{ij}}^{\mathrm{f}}\right\rangle$ may be considered as the stress component induced by fabric. Moreover, $\left\langle\sigma_{\mathrm{ij}}^{\mathrm{f}}\right\rangle$ tend to have no effect on the shear induced plastic deformation of granular materials. Similarly, $\left\langle\sigma_{\mathrm{ij}}^{\mathrm{T}}\right\rangle$ may be regarded as the stress associated with pure interparticle friction. For cohesionless materials, $\left\langle\sigma_{\mathrm{ij}}^{\mathrm{T}}\right\rangle$ vanishes when $\left\langle\sigma_{\mathrm{ij}}^{\mathrm{N}}\right\rangle=0$. As a result, one may argue that, when adopting the stress decomposition of Eq. (30), only a part of the deviatoric stress contributes to plastic shear deformation of granular materials.

\section{FAILURE CRITERIA OF ANISOTROPIC GRANULAR MATERIALS}

\section{Stress Invariant Approach}

This section, which establishes the failure criterion by taking into account the effect of fabric, uses the $s-t$ notation with

$$
s=\frac{1}{2}\left(\sigma_{1}+\sigma_{2}\right), \quad t=\frac{1}{2}\left(\sigma_{1}-\sigma_{2}\right)
$$

being the mean effective and the maximum shear stresses for two-dimensional stress conditions.

Consider a macroscopic shear plane within an ensemble of two-dimensional granules. The plane, with the unit normal $\mathbf{v}$, passes though a large number of granules with various orientations of contact normals. The shearing is assumed to occur in the direction of $\mathbf{s}$ and the orientation of the greatest density of the contact normals makes an angle $\theta_{0}$ with the direction of $\mathbf{s}$, as shown in Fig. 3. Given the contact force at any contact point being

$$
\mathbf{f}=f_{\mathrm{n}} \mathbf{n}+f_{\mathrm{t}} \mathbf{t}
$$

At sliding contacts, one has

$$
m_{0}=\tan \varphi_{0}=\left|f_{\mathrm{t}} / f_{\mathrm{n}}\right|
$$

with $\varphi_{0}$ representing the interparticle friction angle. According to Eq. (27), in a coordinate system with $x$ - and $y$-axis being coincident with $\mathbf{s}$ and $\mathbf{v}$, respectively (see Fig. 3), the stress components $\left\langle\sigma_{\mathrm{ij}}^{\mathrm{N}}\right\rangle$ and $\left\langle\sigma_{\mathrm{ij}}^{\mathrm{T}}\right\rangle$ are

$$
\begin{aligned}
\left\langle\sigma_{\mathrm{xx}}^{\mathrm{N}}\right\rangle= & s\left(1+\frac{\bar{\omega}}{2} \cos 2 \theta_{0}\right) ; \quad\left\langle\sigma_{\mathrm{yy}}^{\mathrm{N}}\right\rangle=s\left(1-\frac{\bar{\omega}}{2} \cos 2 \theta_{0}\right) ; \\
& \left\langle\tau_{\mathrm{xy}}^{\mathrm{N}}\right\rangle=\frac{s}{2} \bar{\omega} \sin 2 \theta_{0}
\end{aligned}
$$




$$
\begin{aligned}
&\left\langle\sigma_{\mathrm{xx}}^{\mathrm{T}}\right\rangle= \frac{m_{0} \mathrm{~S}}{2} \bar{\omega} \sin 2 \theta_{0} ;\left\langle\sigma_{\mathrm{yy}}^{\mathrm{T}}\right\rangle=-\frac{m_{0} \mathrm{~S}}{2} \bar{\omega} \sin 2 \theta_{0} ; \\
&\left\langle\tau_{\mathrm{xy}}^{\mathrm{T}}\right\rangle=-m_{0} \mathrm{~s}\left(1+\frac{\bar{\omega}}{2} \cos 2 \theta_{0}\right)
\end{aligned}
$$

respectively. It should be noted that $\sigma_{\mathrm{xx}}$ and $\tau_{\mathrm{xy}}$ are in fact the normal and shear traction components on the failure plane.

For isotropic materials with $\bar{\omega}=0$,

$$
\begin{gathered}
\left\langle\sigma_{\mathrm{xx}}^{\mathrm{N}}\right\rangle=\left\langle\sigma_{\mathrm{yy}}^{\mathrm{N}}\right\rangle=s ; \quad\left\langle\tau_{\mathrm{xy}}^{\mathrm{N}}\right\rangle=0 \\
\left\langle\sigma_{\mathrm{xx}}^{\mathrm{T}}\right\rangle=\left\langle\sigma_{\mathrm{yy}}^{\mathrm{T}}\right\rangle=0 ; \quad\left\langle\tau_{\mathrm{xy}}^{\mathrm{T}}\right\rangle=-m_{0} \mathrm{~S}
\end{gathered}
$$

which means that the normal components of contact forces do not induce shear stress while the tangential components of contact forces have no effect on either the normal stress on a given plane or the spherical component of the stress tensor. The comparison of Eq. (36) with Eq. (38) and Eq. (37) with Eq. (39) indicates that the contribution of fabric on stresses of anisotropic materials can be expressed as

$$
\begin{aligned}
& \left\langle\Delta \sigma_{\mathrm{xx}}^{\mathrm{N}}\right\rangle=\frac{s}{2} \bar{\omega} \cos 2 \theta_{0} ; \quad\left\langle\Delta \sigma_{\mathrm{yy}}^{\mathrm{N}}\right\rangle=-\frac{s}{2} \bar{\omega} \cos 2 \theta_{0} ; \\
& \left\langle\Delta \tau_{\mathrm{xy}}^{\mathrm{N}}\right\rangle=\frac{s}{2} \bar{\omega} \sin 2 \theta_{0} \\
& \left\langle\Delta \sigma_{\mathrm{xx}}^{\mathrm{T}}\right\rangle=\frac{m_{0} \mathrm{~S}}{2} \bar{\omega} \sin 2 \theta_{0} ; \quad\left\langle\Delta \sigma_{\mathrm{yy}}^{\mathrm{T}}\right\rangle=\frac{m_{0} \mathrm{~S}}{2} \bar{\omega} \sin 2 \theta_{0} ; \\
& \left\langle\Delta \tau_{\mathrm{xy}}^{\mathrm{T}}\right\rangle=-\frac{m_{0} \mathrm{~S}}{2} \bar{\omega} \cos 2 \theta_{0}
\end{aligned}
$$

The deviatoric stress invariant $t^{\mathrm{T}}$ of $\left\langle\sigma_{\mathrm{ij}}^{\mathrm{T}}\right\rangle$, which represents the contribution of tangential contact forces to the stress tensor, is then

$$
\begin{aligned}
t^{\mathrm{T}} & =\sqrt{\left(\frac{\left\langle\sigma_{\mathrm{xx}}^{\mathrm{T}}\right\rangle-\left\langle\sigma_{\mathrm{yy}}^{\mathrm{T}}\right\rangle}{2}\right)^{2}+\left(\left\langle\tau_{\mathrm{yx}}^{\mathrm{T}}\right\rangle\right)^{2}} \\
& =-m_{0} \mathrm{~s} \sqrt{1+\bar{\omega} \cos 2 \theta_{0}+\left(\frac{\bar{\omega}}{2}\right)^{2}}
\end{aligned}
$$

According to Eq. (30), the deviatoric stress invariant of $\left\langle\sigma_{\mathrm{ij}}^{\mathrm{T}}\right\rangle$ can be alternatively calculated by

$$
\left(t^{\mathrm{T}}\right)^{2}=\frac{1}{2} s_{\mathrm{ij}}^{\mathrm{T}} s_{\mathrm{ij}}^{\mathrm{T}}=\frac{1}{2} s_{\mathrm{ij}} s_{\mathrm{ij}}-\frac{1}{2} s s_{\mathrm{ij}} \omega_{\mathrm{ij}}+\frac{1}{4} s^{2}\left(\frac{1}{2} \omega_{\mathrm{ij}} \omega_{\mathrm{ij}}\right)
$$

Referring to Fig. 4, for biaxial stress conditions, the non-coaxiality angle between the stress and fabric tensors is $\delta+\pi / 2$ with $\delta$ being the angle between the bedding plane and the direction of the major principal stress $-\sigma_{1}$. If one further defines the Mohr-Coulomb friction angle $\varphi$ via

$$
-\frac{t}{s}=\sin \varphi
$$

it follows that

$$
\left(\frac{t^{\mathrm{T}}}{s}\right)^{2}=(\sin \varphi)^{2}-\bar{\omega} \cos 2 \delta \sin \varphi+\frac{1}{4} \bar{\omega}^{2}
$$

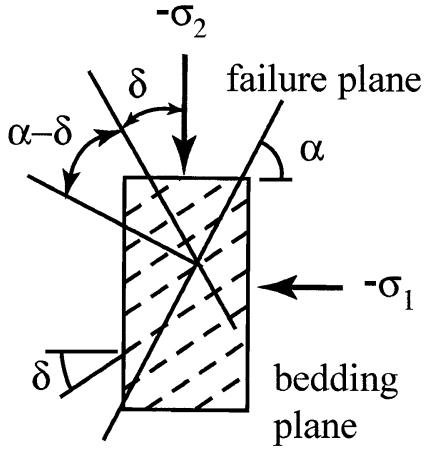

Fig. 4. Bedding plane and failure plane in biaxial compression

$$
\begin{aligned}
\sin ^{2} \varphi= & m_{0}^{2}\left[1+\bar{\omega} \cos 2 \theta_{0}+\left(\frac{\bar{\omega}}{2}\right)^{2}\right] \\
& +\bar{\omega} \cos 2 \delta \sin \varphi-\frac{1}{4} \bar{\omega}^{2}
\end{aligned}
$$

When $\bar{\omega}$ is small enough, the above equation can be simplified as

$$
\sin \varphi \approx m_{0}\left(1+\frac{\bar{\omega}}{2} \cos 2 \theta_{0}\right) \pm \frac{\bar{\omega}}{2} \cos 2 \delta
$$

As illustrated in Fig. 4, for biaxial stress conditions, the angle between the major principal direction of fabric and the sliding direction is

$$
\theta_{0}=\frac{\pi}{2}+\alpha-\delta
$$

with $\alpha$ being the angle between the macroscopic failure plane and the direction of the major principal stress $\sigma_{1}(<0)$. It should be noted that, according to MohrCoulomb failure criterion, $\alpha$ is usually expressed as $\alpha=\pi$ $/ 4+\varphi / 2$.

Equation (47) may also be written as

$$
\sin \varphi \simeq m_{0}\left[1-\frac{\bar{\omega}}{2} \cos 2(\alpha-\delta)\right] \pm \frac{\bar{\omega}}{2} \cos 2 \delta
$$

which is consistent with the experimental results of Haruyama and Kitamura (1984) and Miura et al. (1986). Equations (49) and (47) clearly show that the apparent friction angle $\varphi$ is sensitive to the orientations of the bedding plane and the failure plane, as one may expect.

\section{Critical Plane Approach}

In this section, we intend to show an alternative approach, i.e., the critical plane approach, to establish the failure criterion of anisotropic granular materials. Let us consider the plane $a-a$ shown in Fig. 3 and assume that the orientation of the greatest density of the contact normals makes an angle $\theta_{0}$ with this plane. Given the average tangential and normal traction components, $\tau_{\mathrm{v}}$ and $\sigma_{\mathrm{n}}$ respectively, on plane $a-a$

$$
\begin{gathered}
\tau_{\mathrm{v}}=\left\langle\tau_{\mathrm{xy}}^{\mathrm{N}}\right\rangle+\left\langle\tau_{\mathrm{xy}}^{\mathrm{T}}\right\rangle=\frac{s}{2} \bar{\omega} \sin 2 \theta_{0}-m_{0} S\left(1+\frac{\bar{\omega}}{2} \cos 2 \theta_{0}\right) \\
\sigma_{\mathrm{n}}=\left\langle\sigma_{\mathrm{yy}}^{\mathrm{N}}\right\rangle+\left\langle\sigma_{\mathrm{yy}}^{\mathrm{T}}\right\rangle=s\left(1-\frac{\bar{\omega}}{2} \cos 2 \theta_{0}\right)-\frac{m_{0} \mathrm{~S}}{2} \bar{\omega} \sin 2 \theta_{0}
\end{gathered}
$$


it follows according to Eqs. (36) and (37) that

$$
\frac{\tau_{\mathrm{v}}-\left\langle\tau_{\mathrm{xy}}^{\mathrm{N}}\right\rangle}{\sigma_{\mathrm{n}}-\left\langle\sigma_{\mathrm{yy}}^{\mathrm{T}}\right\rangle}=\frac{\left\langle\tau_{\mathrm{xy}}^{\mathrm{T}}\right\rangle}{\left\langle\sigma_{\mathrm{yy}}^{\mathrm{N}}\right\rangle}=-m_{0} A, \quad A=\frac{1+\frac{\bar{\omega}}{2} \cos 2 \theta_{0}}{1-\frac{\bar{\omega}}{2} \cos 2 \theta_{0}}
$$

which relates the average shear and normal components of the traction vector on plane $a-a$ with the degree of anisotropy and the orientation of fabric. $\tau_{\mathrm{v}}$ and $\sigma_{\mathrm{n}}$ on plane $a-a$ can also be calculated by recognizing that

$$
\tau_{\mathrm{v}}=t \sin 2 \alpha, \quad \sigma_{\mathrm{n}}=s-t \cos 2 \alpha
$$

with $\alpha$ being the angle between the plane and the direction of $-\sigma_{1}$ (see Fig. 4). The combination of Eqs. (50) and (51) yields

$$
-\frac{t}{s}=\sin \varphi=\frac{m_{0} A-\frac{\bar{\omega}}{2}\left(1-m_{0}^{2} A\right) \sin 2 \theta_{0}}{\sin 2 \alpha-m_{0} A \cos 2 \alpha}
$$

Given Eq. (48), Eq. (52) may be rewritten as

$$
\sin \varphi=\frac{m_{0} A+\frac{\bar{\omega}}{2}\left(1-m_{0}^{2} A\right) \sin 2(\alpha-\delta)}{\sin 2 \alpha-m_{0} A \cos 2 \alpha}
$$

The orientation of the critical plane $\alpha_{\mathrm{cr}}$ can be determined from

$$
\frac{d \sin \varphi}{d \alpha}=0 \Rightarrow \alpha=\alpha_{\mathrm{cr}}
$$

which corresponds to a statement of "maximum obliquity'. The apparent friction angle at failure and the corresponding failure criterion are readily obtained when $\alpha_{\mathrm{cr}}$ is obtained from Eq. (54). In other words, the orientation of the failure plane satisfies

$\max _{\alpha} F=\max _{\alpha}\left[\sin \varphi-\frac{m_{0} A+\frac{\bar{\omega}}{2}\left(1-m_{0}^{2} A\right) \sin 2(\alpha-\delta)}{\sin 2 \alpha-m_{0} A \cos 2 \alpha}\right]=0$

as proposed by Pietruszczak and Mroz (2001).

For isotropic materials with $\bar{\omega}=0$, Eqs. (54) and (52) yield $\alpha=\pi / 4+\varphi / 2$ and $-t / s=\sin \varphi_{0}$, respectively, indicating that the classical Mohr-Coulomb failure criterion is recovered.

\section{Special Cases}

When neglecting $\left\langle\tau_{\mathrm{xy}}^{\mathrm{N}}\right\rangle$, which is the shear stress induced by fabric and the normal component of contact force, and $\left\langle\sigma_{\mathrm{yy}}^{\mathrm{T}}\right\rangle$, the normal stress induced by fabric and the tangential component of contact force, Eq. (50) reduces to

$$
\begin{aligned}
\tan \varphi_{\text {local }} & =\left|\frac{\tau_{\mathrm{v}}}{\sigma_{\mathrm{n}}}\right|=m_{0} \frac{1+\frac{\bar{\omega}}{2} \cos 2 \theta_{0}}{1-\frac{\bar{\omega}}{2} \cos 2 \theta_{0}} \\
& \simeq m_{0}\left(1+\bar{\omega} \cos 2 \theta_{0}\right)
\end{aligned}
$$

This relation is the same as the directional distribution function assumed by Pietruszczak and Mroz (2001). In other words, Pietruszczak and Mroz's model may be regarded as a special case of the proposed critical plane approach when one assumes that the normal and tangential interparticle contact forces have no contributions to the tangential and normal tractions on the failure plane via particle arrangements, i.e., $\left\langle\tau_{\mathrm{xy}}^{\mathrm{N}}\right\rangle=\left\langle\sigma_{\mathrm{yy}}^{\mathrm{T}}\right\rangle=0$.

If only $\left\langle\tau_{\mathrm{xy}}^{\mathrm{N}}\right\rangle$ is taken into account and the normal traction on a failure plane is assumed to be independent of fabric, Eq. (50) simplifies to the failure condition proposed by Nemat-Nasser (2000)

$$
f=\tau_{\mathrm{v}}+\frac{s}{2} \sin 2 \varphi_{0}+\frac{s}{2} \bar{\omega} \sin 2 \theta_{0}=0
$$

which indicates that Nemat-Nasser's failure condition is also a special case of the proposed critical plane approach. Using the stress decomposition of Eq. (30), Eq. (56) becomes

$$
f=t^{\mathrm{T}} \sin 2 \theta+\frac{s}{2} \sin 2 \varphi_{0}
$$

with $t^{\mathrm{T}}=\left(s_{\mathrm{ij}}^{\mathrm{T}} S_{\mathrm{ij}}^{\mathrm{T}} / 2\right)^{1 / 2}$. Nemat-Nasser suggests that failure occurs on planes for which Eq. (57) attain its maximum value when

$$
t^{\mathrm{T}}=-s \sin \varphi_{0}
$$

If we consider Eq. (45), the failure criterion for biaxial stress conditions proposed by Nemat-Nasser (2000) may be expressed as

$$
\sin ^{2} \varphi=\sin ^{2} \varphi_{0}+\bar{\omega} \sin \varphi \cos 2 \delta-\frac{1}{4} \bar{\omega}^{2}
$$

Moreover, according to Nemat-Nasser (2000), the failure planes are orientated symmetrically about the major principal stress $-\sigma_{1}$, making an angle of $\pi / 4+\varphi_{0} / 2$ with the direction of $-\sigma_{1}$. Comparing Eq. (59) with Eq. (46), one finds that Eq. (59) is a particular form of Eq. (46) when Eq. (42) takes the form of

$$
t^{\mathrm{T}}=\left(\frac{1}{2} s_{\mathrm{ij}}^{\mathrm{T}} s_{\mathrm{ij}}^{\mathrm{T}}\right)^{1 / 2}=-m_{0} \mathrm{~S}
$$

with $m_{0}=\sin \varphi_{0}$. As a result, one may argue that Eq. (59) is a special case of the proposed stress invariant approach that only takes into account the contribution of fabric on $\left\langle\sigma_{\mathrm{ij}}^{\mathrm{N}}\right\rangle$, that is, the stress partition due to normal components of contact forces. Furthermore, the effect of fabric on $\left\langle\sigma_{\mathrm{ij}}^{\mathrm{T}}\right\rangle$, the stress partition due to tangential components of contact forces, is neglected.

\section{NUMERICAL EXAMPLES}

This section presents numerical examples to illustrate the application of the failure criteria discussed in the previous sections. The discussion here focuses primarily on the dependency of the friction angle at failure on the orientation of bedding plane and the degree of anisotropy. Only granular soils under drained biaxial compression test conditions (see Fig. 4) are considered in these examples. 

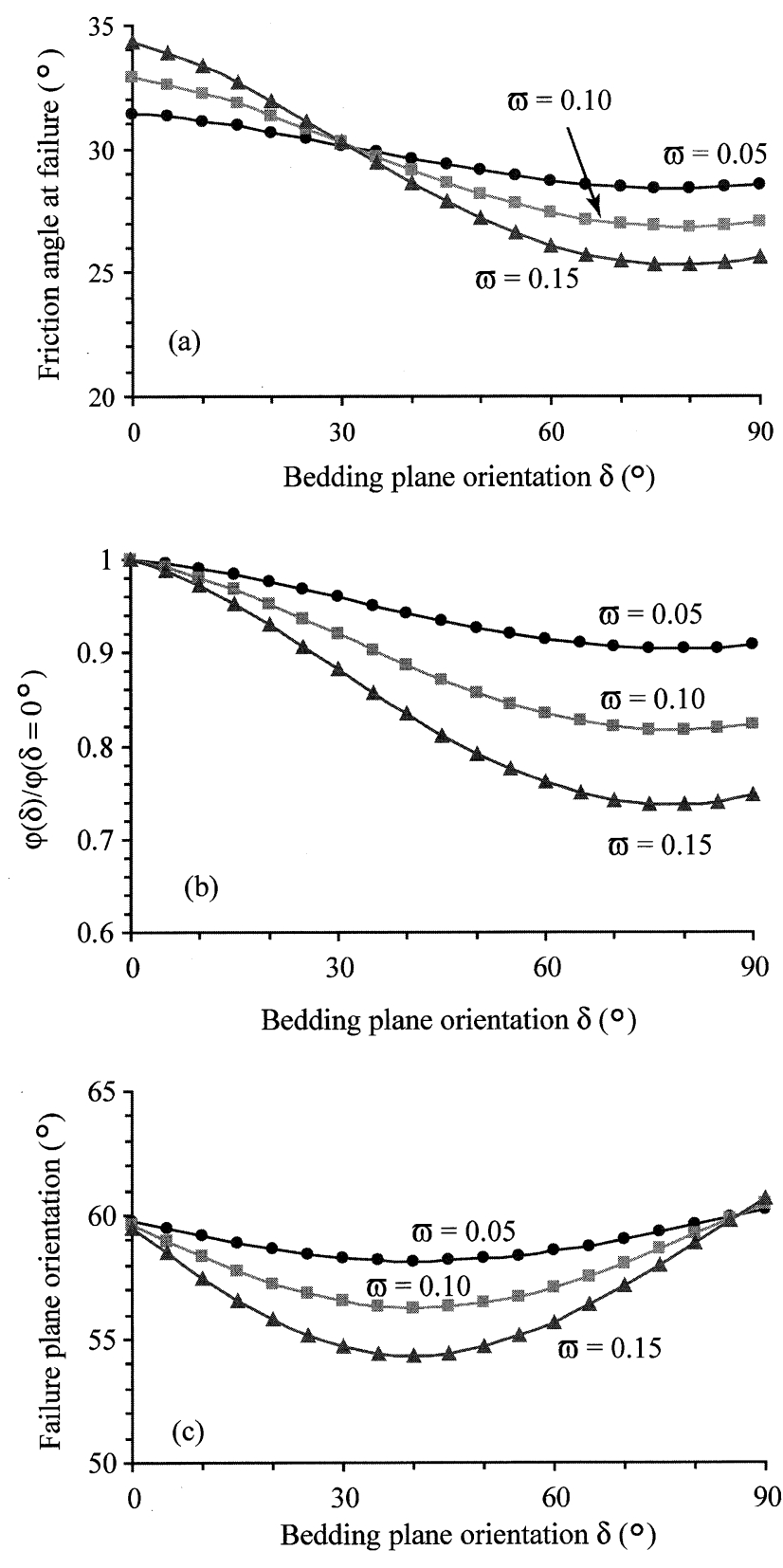

Fig. 5. Calculated apparent friction angle and failure plane orientation: Critical plane approach, $m_{0}\left(=\tan \varphi_{0}\right)=0.577$

Influence of Bedding Plane Angle and the Degree of Anisotropy: Critical Plane Approach

Figure 5 presents the variation of friction angle at failure and the orientation of failure plane calculated from the critical plane approach for a granular material with $m_{0}=0.577$, which corresponds to an interparticle friction of $30^{\circ}$ (see Eq. 35). The second invariant of tensor $\omega$ is chosen as $\bar{\omega}=0.05,0.1$ and 0.15 to describe the effect of the degree of anisotropy. As shown in Fig. 5(a), for a given value of $\bar{\omega}$, the apparent friction angle is the largest for the horizontal bedding plane. With an increase of the orientation angle, $\delta$, of the bedding plane, the friction angle tends to decrease and reaches a minimum at a bedding angle $\delta_{\text {cr }}$ of $\sim 75^{\circ}$. Moreover, the maximum apparent friction angle at $\delta=0^{\circ}$ tends to
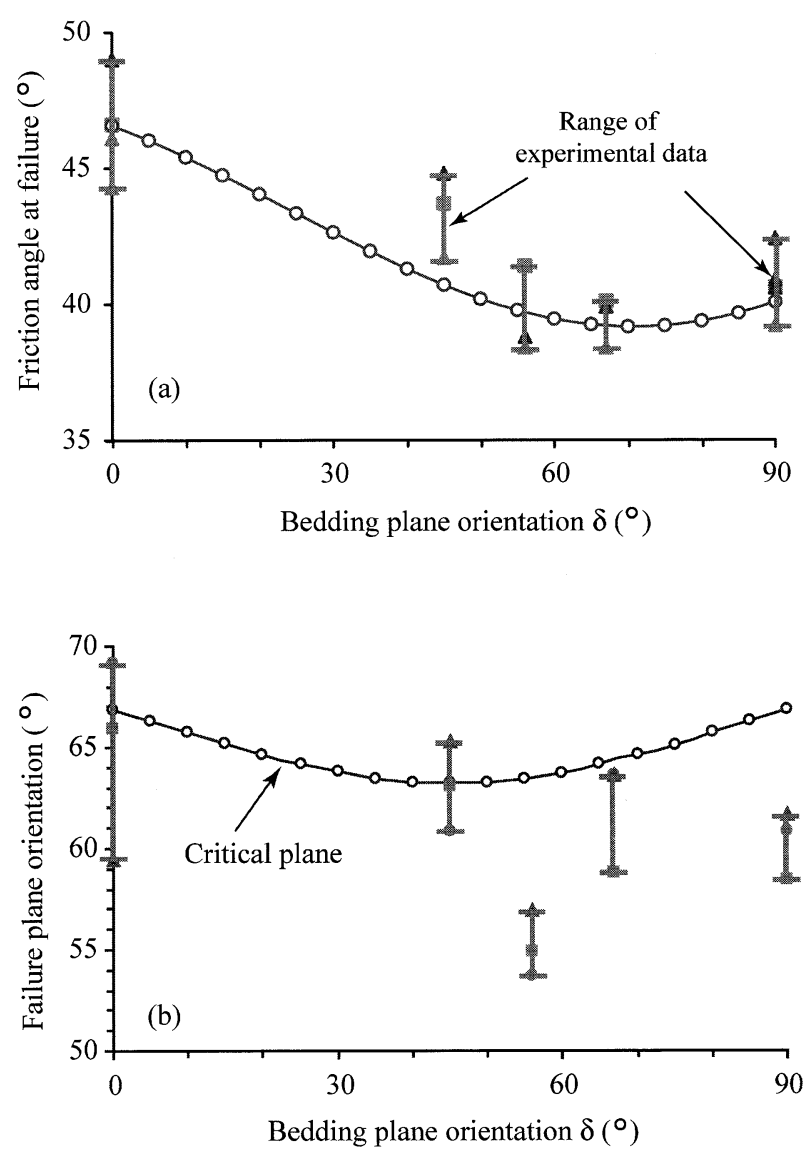

Fig. 6. Calculated apparent friction angle and failure plane orientation: Critical plane approach, $m_{0}\left(=\tan \varphi_{0}\right)=0.95, \bar{\omega}=0.15$

increase with $\bar{\omega}$. The difference between the maximum and the minimum apparent friction angles increases with the degree of anisotropy, which is quantified by $\bar{\omega}$, as one would expect. This can be seen more clearly from Fig. 5(b) that presents the variation of $\varphi(\delta) / \varphi\left(\delta=0^{\circ}\right)$, which is the ratio between friction angles at any bedding angle $\delta$ and that at $\delta=0^{\circ}$. One observes that the reduction of $\varphi(\delta) / \varphi\left(\delta=0^{\circ}\right)$ induced by bedding orientation highly depends on the degree of anisotropy as well as the interparticle friction. For this particular example, the decrease of $\varphi(\delta)$ is as high as $\sim 26 \%$ for $\bar{\omega}=0.15$. The results are qualitatively in agreement with the experimental data reported by, for example, Oda et al. (1978), Tatsuoka et al. (1986) and Tatsuoka et al. (1990).

Figure 5(c) shows the influence of bedding plane orientation and the degree of anisotropy on the orientation of the failure plane. Under biaxial compression, failure occurs along steeper failure planes when the bedding plane is horizontal or vertical, while a shallower failure plane is obtained when the bedding plane angle is about $40^{\circ}$. The inclination angle of the failure plane, $\alpha$, tends to decrease with increasing $\bar{\omega}$. However, the degree of anisotropy has less effect on $\alpha$ when the bedding plane approaches horizontal or vertical.

The validity of the proposed critical plane approach is further checked by comparing model predictions with experimental results. Figure 6 compares the experimental 


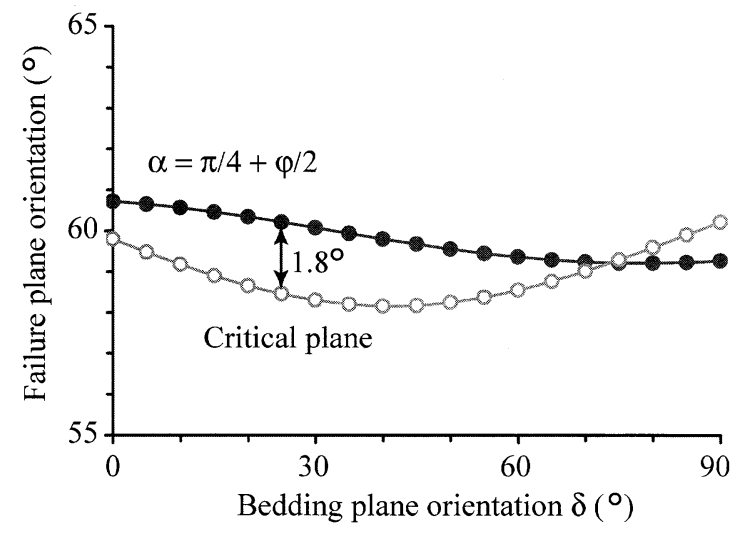

(a) $\bar{\varpi}=0.05, \mathrm{~m}_{\mathrm{o}}\left(=\tan \varphi_{\mathrm{o}}\right)=0.577$

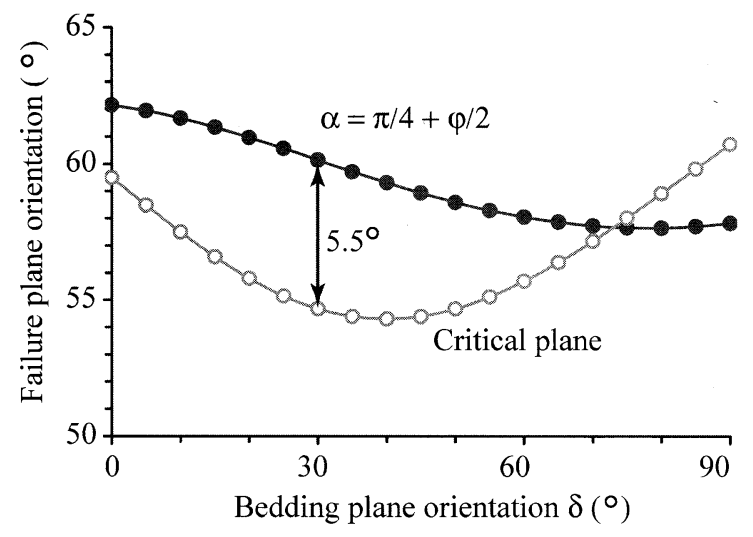

(b) $\varpi=0.15, \mathrm{~m}_{\mathrm{o}}\left(=\tan \varphi_{\mathrm{o}}\right)=0.577$

Fig. 7. Orientation of failure planes: Comparison between the critical plane approach and classical Mohr-Coulomb Theory

data of Toyoura sand (Tatsuoka et al., 1990) with the apparent failure friction angle calculated from the proposed critical plane approach, assuming $\bar{\omega}=0.15$ and $m_{0}=0.95$ (or $\left.\varphi_{0}=43.5^{\circ}\right)$. It is clearly shown that the proposed critical plane approach predicts the correct trend of the variation of friction angle with the bedding plane orientation. The theoretical failure plane orientation, however, agrees with experimental data only when the bedding plane angle is less than $\sim 45^{\circ}$. On the other hand, the model tends to over predict the angle of failure plane $\alpha$. One should recognize that the experimental value of the failure plane orientation is more subjective than the theoretical value, as it is based on observation. Moreover, particle spins that occur during deformation, which may have substantial effects on failure plane orientations particularly at large bedding plane angles, are not taken into account in the model at this point. Consequently, it should not be surprising that the two deviate as shown in Fig. 6(b).

Figure 7 further compares the orientation of the failure plane calculated from the critical plane approach and that obtained from Mohr-Coulomb's theory in which the failure planes make an angle of

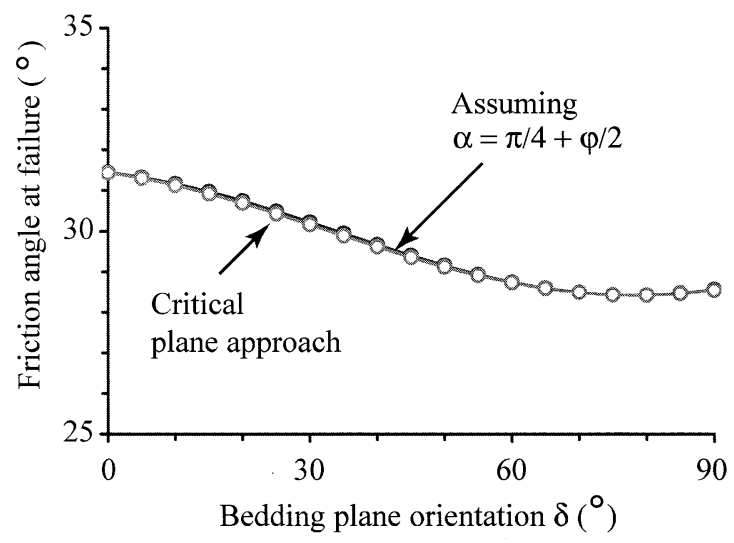

(a) $\varpi=0.05, \mathrm{~m}_{\mathrm{o}}\left(=\tan \varphi_{\mathrm{o}}\right)=0.577$

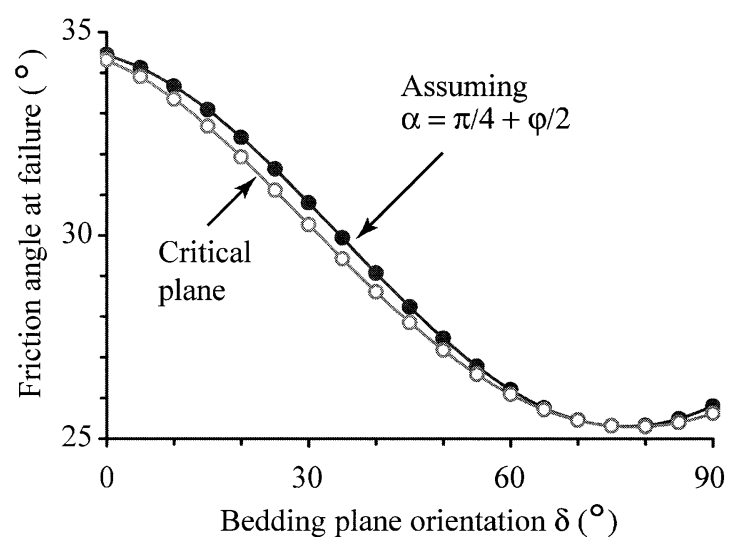

(b) $\varpi=0.15, \mathrm{~m}_{\mathrm{o}}\left(=\tan \varphi_{\mathrm{o}}\right)=0.577$

Fig. 8. Sensitivity of the apparent friction angle with respect to the orientation of failure plane

$$
\alpha_{\mathrm{MC}}= \pm(\pi / 4+\varphi / 2)
$$

with the direction of $-\sigma_{1}$. The results in Fig. 7 show that Eq. (61) may not be applicable to anisotropic materials. When $m_{0}=0.577$, the maximum differences between $\alpha_{\mathrm{MC}}$ and $\alpha_{\text {cr }}$ obtained from the proposed critical plane approach are $1.8^{\circ}$ and $5.5^{\circ}$ for $\bar{\omega}=0.05$ and 0.15 , respectively.

\section{Sensitivity of the Orientation of Failure Planes}

The sensitivity of the friction angle with respect to the orientation of failure planes is investigated next. Substituting Eq. (61) into Eq. (52) gives the friction angle when the failure plane orientation is assumed to be $\alpha_{\mathrm{MC}}$. Figure 8 shows that, when $\bar{\omega}=0.05$ and 0.15 , the assumption $\alpha=\alpha_{\mathrm{MC}}$ induces an increase of $0.24^{\circ}$ and $0.54^{\circ}$ in the apparent friction angle, respectively. Referring to Fig. 7, the maximum differences between $\alpha_{\mathrm{MC}}$ and $\alpha_{\mathrm{cr}}$ are $1.8^{\circ}$ and $5.5^{\circ}$, respectively. This implies that the apparent friction angle is not very sensitive to the orientation of failure plane. In other words, the failure plane orientation may vary in a certain range while the apparent friction angle does not change much, as shown in Fig. 7. These observations can also explain the discrepancy between the 


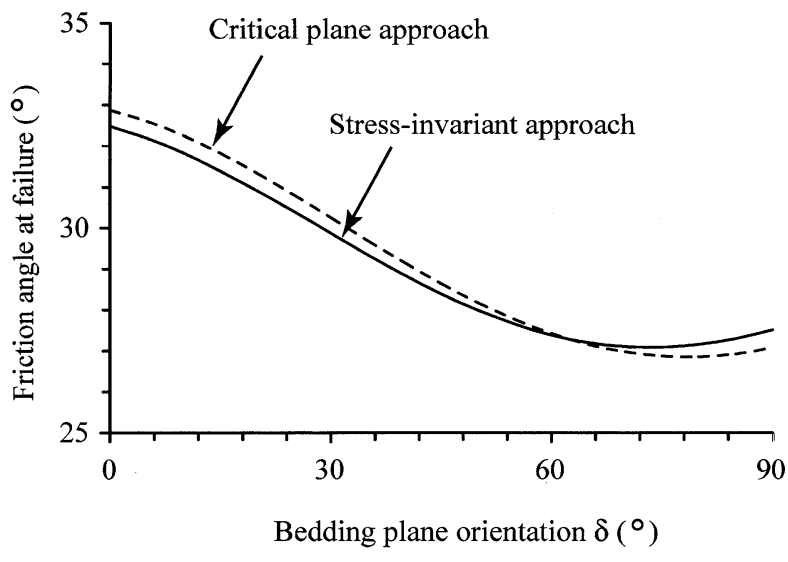

(a) $\varpi=0.10, \mathrm{~m}_{\mathrm{o}}=0.577$ (or $\varphi_{\mathrm{o}}=30^{\circ}$ )

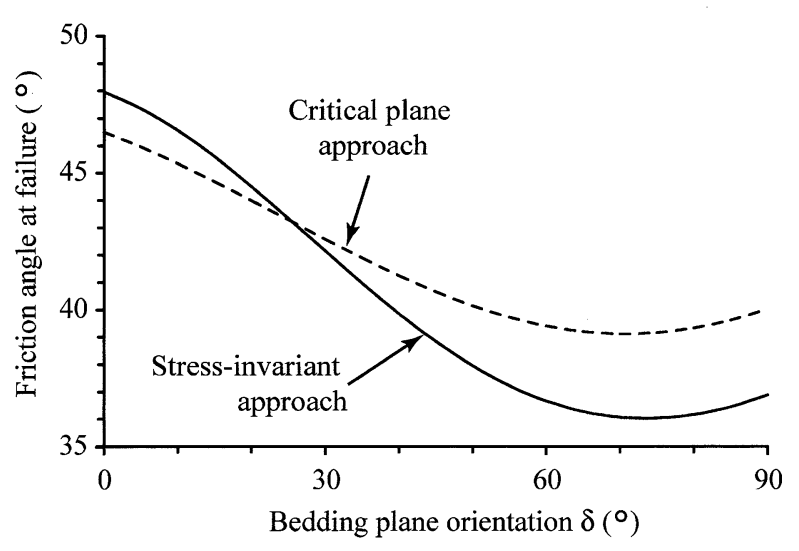

(b) $\bar{\sigma}=0.15, \mathrm{~m}_{\mathrm{o}}=0.95\left(\right.$ or $\left.\varphi_{\mathrm{o}}=43.5^{\circ}\right)$

Fig. 9. Comparison of stress-invariant and critical plane approaches

calculated and experimental failure plane orientations shown in Fig. 6(b).

\section{Comparison of Stress Invariant and Critical Plane Approaches}

Figure 9 compares the calculated apparent friction angles from the critical plane and the stress invariant approaches for various bedding plane orientations. When the interparticle friction angle $\varphi_{0}=30^{\circ}$ and $\bar{\omega}=0.1$, the stress invariant and critical plane approaches predict similar friction angles. On the other hand, for granular materials with $\varphi_{0}=43.5^{\circ}$, a substantial discrepancy in the apparent friction angles is observed, indicating that the utilization of stress invariants to describe the failure condition may undermine the effect of fabric on the failure of anisotropic granular materials.

The difference between the critical plane and the stress invariant approaches is attributed to the manner in which the effect of fabric is taken into account. In the critical plane approach, the failure criterion is checked in terms of normal and tangential components of the traction vector on each potential failure plane and the critical one is obtained from the maximization of the failure function. On the other hand, the stress invariant approach, which assumes that failure occurs along the plane with $\alpha=\pi / 4+\varphi / 2$, focuses on the relation between stress invariants of an ensemble of grains within a representative volume element. Moreover, the shear resistance is considered consisting of two components due to fabric and tangential interparticle forces, respectively. Referring to Eq. (30), the influence of fabric on the spherical stress component of the stress tensor may vanish when the spherical stress is employed to establish a failure criterion in the stress invariant approach. This implies that some microscopic features of the granular material may not be captured when using stress invariants that are average quantities of a representative volume element. Consequently, one observes that the difference between the results obtained from the critical plane approach and the stress invariant approach varies with the degree of anisotropy and the interparticle friction angle, as confirmed by the results of Fig. 9 .

\section{Comparison with Other Models}

Figures 10 and 11 compare the friction angle at failure and the orientation of critical failure planes determined by different methods. When comparing the proposed critical plane approach with Pietruszczak and Mroz's model, given $\varphi_{0}=30^{\circ}$ (or $m_{0}=0.577$ ), one observes remarkable differences in both the friction angle at failure for materials with high degree of anisotropy (i.e. $\bar{\omega}=0.15$ in Figs. 10(c) and (d)). For materials of minor anisotropy with $\bar{\omega}=0.05$, the discrepancy between the values of friction angles decreases. For Pietruszczak and Mroz's model, the minimum apparent friction angle at various degrees of anisotropy appears at a bedding plane angle $\delta$ of about $60^{\circ}$, while the smallest orientation angle of the failure plane is obtained at $\delta \simeq 30^{\circ}$. These values of $\delta$ are different with those obtained from the proposed critical plane approach in which $\delta \simeq 75^{\circ}$ and $40^{\circ}$ for the minimum apparent friction angle and the smallest orientation angle of the failure plane, respectively. In addition to the degree of anisotropy, the interparticle friction angle also contributes to the differences between these two approaches. As shown in Fig. 11, when $\varphi_{0}=43.5^{\circ}$, similar results in terms of the apparent friction angle and the failure plane orientation are observed for both approaches, even when $\bar{\omega}=0.15$.

The differences between the proposed critical plane approach and that of Pietruszczak and Mroz can be explained as follows. Referring to Eqs. (51) and (55), the directional distribution of friction angle proposed by Pietruszczak and Mroz (2001) is recovered from Eq. (51) when $\left\langle\tau_{x y}^{\mathrm{N}}\right\rangle$ and $\left\langle\sigma_{\mathrm{yy}}^{\mathrm{T}}\right\rangle$ are neglected. Since $\left\langle\tau_{\mathrm{xy}}^{\mathrm{N}}\right\rangle$ reflects the combined effect of fabric and the normal contact forces on the shear stress while $\left\langle\sigma_{\mathrm{yy}}^{\mathrm{T}}\right\rangle$ quantifies the contribution of shear contact forces on the normal stress owing to fabric, one would expect that when the contribution of fabric to shear resistance of granular soils is much smaller than that of interparticle frictions, the proposed critical plane approach expressed by Eq. (51) would approach the model proposed by Pietruszczak and Mroz. However, for highly anisotropic materials with low interparticle friction angle $\varphi_{0}$, a significant difference may exist, as 


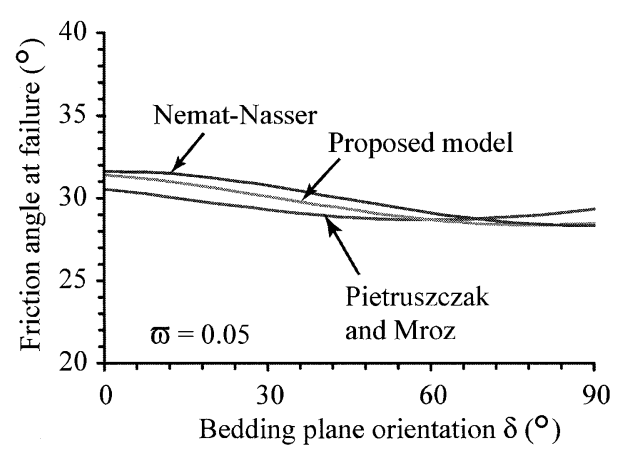

(a)

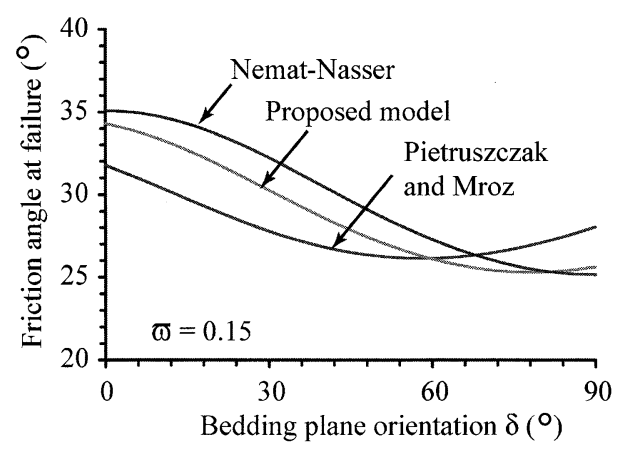

(c)

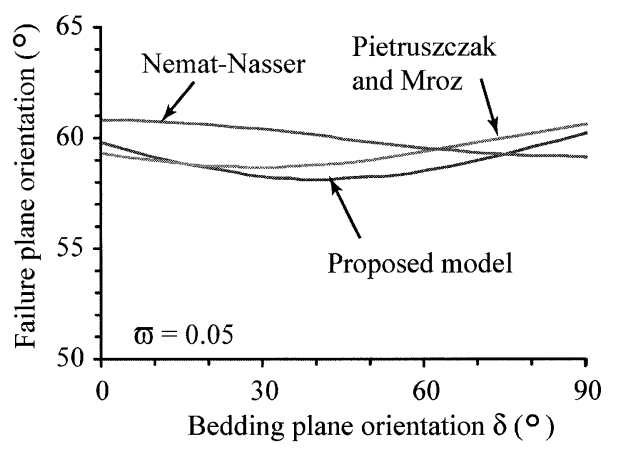

(b)

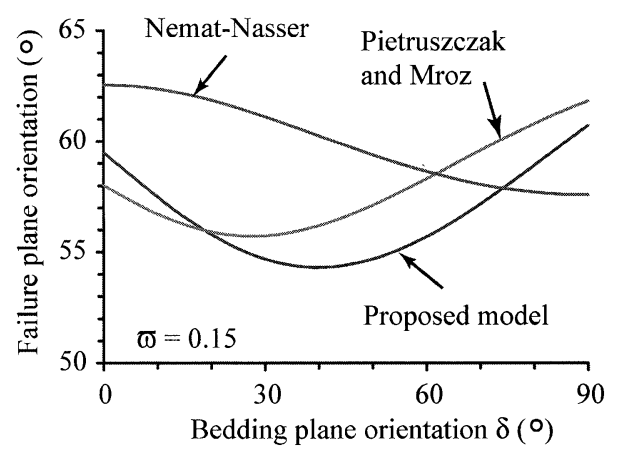

(d)

Fig. 10. Calculated apparent friction angle and failure plane orientation at $m_{0}\left(=\tan \varphi_{0}\right)=0.577$ : Critical plane approach

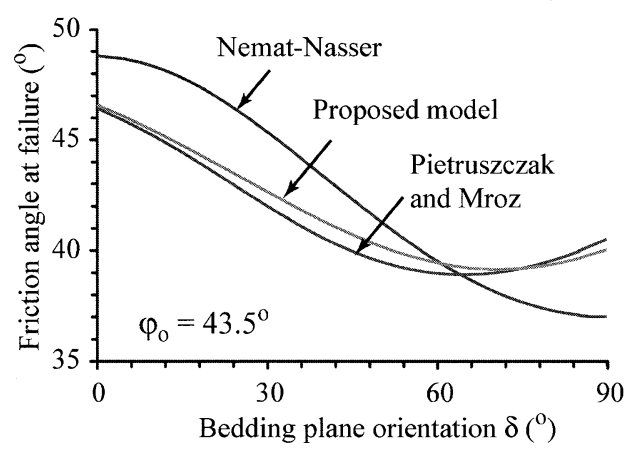

(a)

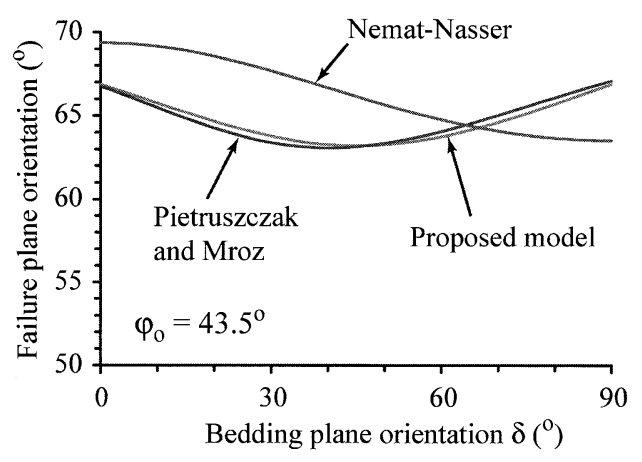

(b)

Fig. 11. Calculated apparent friction angle and failure plane orientation at $\bar{\omega}=\mathbf{0 . 1 5}$ shown in Fig. 10. One also observes that the increase in the apparent friction angle at higher bedding plane angles is captured by both Pietruszczak and Mroz's model and the proposed approach.

Figures 10 and 11 also present the apparent friction angle at failure and the orientation of critical failure planes determined using the method proposed by NematNasser (2000). In general, the magnitudes and trends predicted by Nemat-Nasser's model are different from those obtained from the critical plane models.

For shallow bedding planes (e.g., $\delta<70^{\circ}$ ), the apparent friction angle calculated from Nemat-Nasser's method is larger than that calculated from either the proposed critical plane approach or Pietruszczak and Mroz's model. Moreover, Nemat-Nasser's method predicts a monotonic decrease of the apparent friction angle with respect to bedding plane orientations, which is inconsistent with the experimental results shown in Fig. 6. In addition, the differences among the apparent friction angles calculated from different models vary with the interparticle friction angle $\varphi_{0}$ and the degree of anisotropy. Since Nemat-Nasser only takes into account the effect of $\left\langle\tau_{\mathrm{xy}}^{\mathrm{N}}\right\rangle$ for anisotropic granular soils and neglects the influence of fabric on $\left\langle\tau_{\mathrm{xy}}^{\mathrm{T}}\right\rangle,\left\langle\sigma_{\mathrm{yy}}^{\mathrm{N}}\right\rangle$ and $\left\langle\sigma_{\mathrm{yy}}^{\mathrm{T}}\right\rangle$, one would expect that when the degree of anisotropy is not significant, the friction angle calculated from the proposed model should approach Nemat-Nasser's model. It should be noted that the angle between the failure plane and the direction of $-\sigma_{1}$ is assumed to be $\pi / 4+\varphi_{\mu} / 2$ in Nemat-Nasser's method. 


\section{CLOSING REMARKS}

This paper presents the formulation of anisotropic failure criteria for granular soils via a micromechanical analysis. By considering the micromechanics, the stress tensor is decomposed into three components that allow the specification of anisotropic strength related to the spatial distribution of contact normals. The resulting failure criterion can be regarded as an extension of classical isotropic criteria taking into account fabric effects. The proposed critical plane approach takes into account the effect of fabric and interparticle contact forces, including the contribution of normal contact force components to shear stresses, $\left\langle\tau_{\mathrm{xy}}^{\mathrm{N}}\right\rangle$, and tangential contact force components to normal stresses $\left\langle\sigma_{\mathrm{yy}}^{\mathrm{T}}\right\rangle$. However, the critical plane is determined from a maximization procedure, which would be inconvenient when it is implemented in numerical analysis. For practical applications, this inconvenience might be eliminated by assuming that failure occurs on the plane with the maximum stress obliquity if the material is not highly anisotropic. The models proposed by Pietruszczak and Mroz (2001) and Nemat-Nasser (2000) may be considered as special cases of the proposed critical plane approach, depending on which contribution of interparticle contact forces to the traction vector on the failure plane are neglected. In the stress invariant approach, the mean stress does not involve the traceless $\left\langle\sigma_{\mathrm{ij}}^{\mathrm{T}}\right\rangle$, that is, the stress partition due to tangential components of contact forces, and some microscopic features of granular materials may not be captured.

The predicted friction angle at failure based on the proposed stress-invariant approach may be close to that obtained from the critical plane method, depending on the degree of anisotropy and the interparticle friction angle. This approach can be used to replace the critical plane approach for materials with low interparticle friction. On the other hand, a significant difference in the predicated apparent friction angle may be obtained for materials with high interparticle friction.

In closing, it has been shown that the decomposition of the stress tensor into three components provides valuable insights when considering the effect of fabric and yields more realistic failure criteria for anisotropic granular soils.

\section{ACKNOWLEDGEMENTS}

Funding provided by the Natural Science and Engineering Research Council of Canada is gratefully acknowledged.

\section{REFERENCES}

1) Biarez, J. and Wiendieck, K. (1963): La comparaison qualitative entre l'anisotropie mécanjque et l'anisotropie de structure milieux pulverulents, C. R. Acad. Sci., 256, 1217-1220.

2) Boehler, J. P. (1987): Application of Tensor Functions in Solid Mechanics, Springer, Wien, Chapters 1-7.

3) Cambou, B., Dubujet, P., Emeriault, F. and Sidoroff, F. (1995):
Homogenization for granular materials, Eur. J. Mech. A, Solids, 14 (2), 225-276.

4) Chang, C. S. (1993): Micromechanical modelling of deformation and failure for granulates with frictional contacts, Mech. Mater., 16, 13-24.

5) Chang, C. S. and Misra, A. (1988): Packing structure and mechanical properties of granulates, J. Geotech. Eng., 116 (5), 1077-1093.

6) Cowin, S. C. (1985): The relationship between the elasticity tensor and the fabric tensor, Mech. Materials, 4, 137-147.

7) Emeriault, F. and Chang, C. S. (1997): Interparticle forces and displacements in granular materials, Computers and Geotechnics, 20 (3-4), 223-244.

8) Haruyama, M. and Kitamura, R. (1984): Anisotropic deformationstrength properties of soft sedimentary rock "Shirasu" originated from pyroclastic flows, Soils and Foundations, 24 (4), 84-94.

9) Hoek, E. and Brown, E. T. (1980): Empirical strength criterion for rock masses, J. Geotech. Eng. Div., ASCE, 106 (GT9), 1013-1035.

10) Kanatani, K. (1984): Distribution of directional data and fabric tensors, Int. J. Eng. Sci., 22, 149-164.

11) Kuo, C. Y. and Frost, J. D. (1996): Uniformity evaluation of cohesionless specimens using digital image analysis, J. Geotech. Eng., 122 (5), 390-396.

12) Landau, L. D. and Lifshitz, E. M. (1959): Statistical Physics: Course of Theoretical Physics 5, Oxford Pergamon Press, 1-2.

13) Miura, K., Toki, S. and Mirua, S. (1986): Deformation prediction for anisotropic sand during rotation of principal stress axes, Soils and Foundations, 26 (3), 42-56.

14) Muhunthan, B., Chameau, J. L. and Masad, E. (1996): Fabric effects on the yield behaviour of soils, Soils and Foundations, 36 (3), 85-97.

15) Nemat-Nasser, S. (2000): A micromechanically-based constitutive model for frictional deformation of granular materials, J. Mech. Phys. Solids, 48, 1541-1563.

16) Nova, R. (1980): The failure of transversely anisotropic rocks in triaxial compression, Int. J. Rock Mechanics, Mining Sci. \& Geomech. Abstracts, 17, 325-332.

17) Oda, M. (1989): Yield function for soil with anisotropic fabric, $J$. Eng. Mech. Div., 115 (1), 89-104.

18) Oda, M. and Konishi, J. (1974): Microscopic deformation mechanism of granular material in simple shear, Soils and Foundations, 14 (4), 25-38.

19) Oda, M., Koishikawa, I. and Higuchi, T. (1978): Experimental study of anisotropic shear strength of sand by plane strain tests, Soils and Foundations, 18 (1), 25-38.

20) Oda, M., Nemat-Nasser, S. and Konishi, J. (1985): Stress-induced anisotropy in granular masses, Soils and Foundations, 25 (3), 85-97.

21) Pietruszczak, S. (1999): On inelastic behaviour of anisotropic frictional materials, Mechanics of Cohesive Frictional Materials, 4, 281-293.

22) Pietruszczak, S. and Mroz, Z. (2001): On failure criteria for anisotropic cohesive-frictional materials, Int. J. Numer. Anal. Methods Geomech., 25, 509-524.

23) Rogers, T. G. (1987): Yield criteria, flow rules, and hardening in anisotropic plasticity, Yielding, Damage, and Failure of Anisotropic Solids (ed. by Boehler, J. P.), Mechanical Engineering Publication Limited, London, 53-79.

24) Rothenburg, L. and Bathurst, R. J. (1989): Micromechanical features of granular assemblies with planar elliptical particles, Géotechnique, 42 (1), 79-95.

25) Satake, M. (1982): Fabric tensor in granular materials, Deformation and Failure of Granular Materials (eds. by Vermeer, P. A. and Luger, H. J.), Rotterdam: Balkema, 63-68.

26) Tatsuoka, F., Sakamoto, M., Kawamura, T. and Fukushima, S. (1986): Strength and deformation characteristics of sand in plane strain compression at extremely low pressures, Soils and Foundations, 26 (1), 65-84.

27) Tatsuoka, F., Nakamura, S., Huang, C. and Tani, K. (1990): Strength anisotropy and shear band direction in plane strain tests of sand, Soils and Foundations, 30 (1), 35-54.

28) Thornton, C. (2000): Numerical simulation of deviatoric shear 
deformation of granular media, Géotechnique, $\mathbf{5 0}$ (1), 43-53.

29) Vaid, Y. P. and Saivathayalan, S. (2000): Fundamental factors affecting liquefaction susceptibility of sands, Can. Geotech. J., 37, 592-606.
30) Walsh, J. B. and Brace, J. F. (1964): A fracture criterion for brittle anisotropic rock, J. Geophysical Research, 69, 3449-3456.

31) Yoshimine, M. and Ishihara, K. (1998): Flow potential of sand during liquefaction, Soils and Foundations, 38 (3), 189-198. 